\title{
Extreme bushfire projections for Australia using a standardised method
}

\author{
Andrew J. Dowdy ${ }^{\mathrm{a}}$, Marcus Thatcher ${ }^{\mathrm{b}}$, Tony Rafter ${ }^{\mathrm{b}}$, Jason Evans ${ }^{\mathrm{c}}$, Hua Ye ${ }^{\mathrm{a}}$, Chun-Hsu Su \\ Christian Stassen ${ }^{a}$, Acacia Pepler ${ }^{a}$, Samuel Bell ${ }^{\mathrm{a}}$ and Peter Steinle ${ }^{\mathrm{a}}$ \\ ${ }^{a}$ Bureau of Meteorology, Docklands, Australia \\ ${ }^{b}$ CSIRO Oceans and Atmosphere, Aspendale, Australia \\ ${ }^{c}$ University of New South Wales, Sydney, Australia \\ Email: andrew.dowdy@bom.gov.au
}

\begin{abstract}
The influence of anthropogenic climate change on extreme bushfire weather in Australia is assessed using a standardised method for projections information. The method steps comprise a review and synthesis of a comprehensive range of factors based on observations, modelling and physical process understanding. The resultant lines of evidence are then used to guide the production of projections data and confidence assessments. Projections are produced based on global climate model output as well as dynamical downscaling data using three regional climate modelling approaches (CCAM, BARPA and NARCliM/WRF). The projections data are calibrated using quantile matching methods trained on observations-based data, with a particular focus on the accurate representation of extremes. The resultant projections data include nationally consistent maps of bushfire weather indices corresponding to the 10-year average recurrence interval (i.e., return period) around the middle of this century (2040-2059), with a focus of the discussion on regions around southern and eastern Australia during summer as needed for some risk assessment applications. The projections data are also available for other seasons and time periods throughout this century, as well as for other metrics of extreme or average conditions. The results for southern and eastern Australia during summer show more dangerous bushfire conditions (high confidence in southern Australia; medium confidence in eastern Australia) attributable to increasing greenhouse gas emissions.
\end{abstract}

Keywords: Bushfires, projections, regional downscaling, hazards, wildfire 


\section{INTRODUCTION}

Recent events such as the Black Summer 2019-2020 highlight the need to consider rare and unprecedented extremes particularly in a changing climate (Dowdy 2020; van Oldenburg et al. 2021). Climate change projections are presented here for extreme fire weather conditions based on applying a standardised method. This method uses a comprehensive range of lines of evidence from physical process understanding, observations, reanalyses and climate modelling. It is designed to be beneficial particularly in cases with many contributing factors and uncertainties (such as for some extremes and for mean rainfall, wind, flood, etc.), including for the selection of projections methods and datasets as well as for confidence assessments. For example, this method can help provide guidance when producing the projections data products on whether a particular modelling approach could be useful to include or not (or perhaps weighted differently within a broader ensemble of datasets). That type of targeted guidance can be used along with other more general sources of guidance relevant to projections data, such as based on broader assessments of models and methods relating to climate change projections (CSIRO and BoM 2015; Thatcher et al. 2021). This standardised method is used here together with a new set of calibrated climate projections for Australia, including using three regional downscaling approaches, with the aim of providing the best-available projections information for dangerous bushfire weather conditions under future climate change. For further details around this method and applications such as presented here see Dowdy et al. (2021).

Here we focus on extremes corresponding to the 10-year average recurrence interval (ARI), representing an event with a return period of 10 years on average (noting that the return period is equal to the reciprocal of the annual probability of exceedance). This included maps of the most likely future projected change in values corresponding to the 10 -year ARI, together with estimates of the $10^{\text {th }}$ and $90^{\text {th }}$ percentile range of plausible 10-year ARI values as a confidence assessment measure. National maps of those quantities are presented here, together with confidence assessment information, based on considering many lines of evidence. The resultant maps and data layers (with supporting confidence assessment information) are intended for a broad range of user groups including in sectors for which extreme bushfires are relevant. The projections are presented for the future climate around the middle of this century, as well as for the historical climate, but are also available for other time periods throughout this century as well as for other metrics of extreme or average conditions throughout Australia (with data available on request).

Bushfires can be considered as a form of compound event given the range of factors that influence their occurrence, including based on the combined influence of many weather factors (from near-surface conditions to higher-level atmospheric processes including convection). Bushfire occurrence is also influenced by other factors including vegetation conditions (such as relating to fuel load and type) and ignition sources (such as associated with human activities or with lightning), some of which can be challenging to model (given current climate modelling capabilities). The primary focus of the analysis is on dangerous weather conditions for bushfire hazards, with other factors also considered to some degree (i.e., relating to vegetation conditions and ignition sources), noting that climate change influence on other components of risk (exposure and vulnerability) are less clear than for the hazard component examined here.

\section{DATA AND METHODS}

Bushfire weather conditions are often represented by indices as a useful way of combining numerous weather conditions known to influence fire behaviour. Examples of such indices include the McArthur Forest Fire Danger Index (FFDI) commonly used in Australia, including as the focus of the analysis presented here, which is based on near-surface humidity, wind speed and temperature as well as rainfall (McArthur 1967). There are also many other examples of similar indices to the FFDI, such as the Fire Weather Index (FWI) that was originally developed in Canada but now widely used throughout the world (van Wagner 1974; Field et al. 2015). Indices have also been developed for grass fires, while noting that grass fires were not identified as a key hazard of interest by stakeholders for this research. Indices are also available for several other fuel types including a multi-index system currently in development for Australia (known as the Australian Fire Danger Rating System: AFDRS). Some indices such as the Continuous-Haines index (C-Haines) are based on conditions at higher levels of the atmosphere that can be useful for indicating risk factors associated with the occurrence of extreme fire events, including very dangerous fires that generate thunderstorms in their fire plumes known as pyrocumulonimbus or pyroCb clouds (Mills \& McCaw 2010; Dowdy and Pepler 2018; Di Virgilio et al. 2019).

Several datasets are available for future projections of values corresponding to 10-year ARI of daily fire weather based on the FFDI. These datasets have all been calibrated using the quantile matching for extremes (QME) method described in Dowdy (2020) which also includes details on how the FFDI was calculated as used here, noting that the FFDI is just used here as a broad-scale indication for aspects of fire weather climatology (i.e., as a useful way to combine several factors known to influence fire behaviour including 
humidity, wind, temperature and accumulated rainfall, noting many other factors such as ignition and fuel conditions). These calibrated projections datasets provide a 16-member ensemble, as follows:

- Dynamical downscaling using the CCAM modelling approach (Thatcher and McGregor 2011) applied to 5 GCMs (ACCESS1-0, CanESM2, GFDL-ESM2M, MIROC5, NorESM1-M);

- Dynamical downscaling using the recently developed BARPA modelling approach (Su et al. 2021) applied here to one GCM (ACCESS1-0 GCM for eastern Australia);

- Dynamical downscaling using the NARCliM modelling approach (Evans et al. 2014) applied to 3 GCMs (ACCESS1-0, ACCESS1-3, CanESM2) with 2 configurations of each (providing 6 ensemble members);

- Calibrated data based on the QME method applied to four GCMs (ACCESS1-0, CNRM-CM5, GFDLESM2M, MIROC5 GCMs).

The standardised method consists of two steps, referred to here as producing the Lines of Evidence Table (Step 1) and then producing the Projections Likelihood Information (Step 2). The Lines of Evidence Tables are provided to document the supporting science details, as well as to help guide the production of the Projections Likelihood Information including the confidence assessment. For the purposes of this study, the Projections Likelihood Information is shown as maps for the most probable change in values corresponding to the 10-year ARI, together with estimates of the $10^{\text {th }}$ and $90^{\text {th }}$ percentile range of plausible change in those 10 -year ARI values (as one measure for providing confidence information).

Step 1 - Produce the Lines of Evidence Table

$>$ Collect a wide range of information on climate change that could be of relevance to consider when populating the Lines of Evidence Table. This information could be obtained from new analyses as well as from a review of existing literature, considering aspects such as observations, reanalyses, model data and physical process understanding. For example, relevant aspects to consider could potentially include analysis of long-term observed trends, model simulations of future climate, uncertainties in observations, uncertainties relating to a modelling approach's ability to simulate physical processes and observed features (such as the seasonal cycle or spatial detail of extremes), as well as the influence of large-scale drivers (e.g., El Niño-Southern Oscillation) in the historical and future projected climates.

$>$ Collate that information into short text summaries on each aspect being considered, with accompanying figures and references provided to support those summaries, aiming for a general balance of evidence based on the available science. The summaries can be grouped into broader categories (e.g., physical processes, historical climate and future climate).

$>$ Use those short text summaries to populate the Lines of Evidence Table. This table contains a different row for each of the different aspects being considered. Key details can be listed on each row including the degree of influence that this aspect has on the variable in the region being considered, as well as what this implies for the direction of projected future change (colour-coded to show either an increase, decrease, little change or increased uncertainty). This is intended as a standardised way to help collate and synthesise a broad range of information.

Step 2 - Produce the Projections Likelihood Information

$>$ For the projected change of interest (e.g., a change from the historical period to a future period in values corresponding to the 10-year ARI), use the Lines of Evidence Table to determine the best available data and methods for estimating a given likelihood measure. For example, likelihood measures could include the most probable projected change, together with estimates of the $10^{\text {th }}$ and $90^{\text {th }}$ percentile range of plausible change. The method to determine the best available estimate for a given likelihood measure may vary between different weather variables of interest (e.g., depending on the degree of confidence in models to simulate relevant physical processes). For example, this variation could include the selection of different datasets and methods (e.g., the use of direct model output or statistical diagnostic methods) or scaling some data differently in a model ensemble.

$>$ For quantities that have a reasonably robust range of evidence, with good agreement between those different lines of evidence (e.g., about two thirds of the Lines of Evidence Table having a consistent sign of future change), model output may be the best option for producing the Projections Likelihood Information, while still considering the uncertainties and strengths/weaknesses of different modelling approaches for helping to guide the production of the data products. For quantities with lower confidence (i.e., more limited evidence and/or lower agreement between lines of evidence), a more qualitative best estimate could be appropriate. For example, in some cases with very high uncertainty the best estimate for the Projections Likelihood Information might simply be 'an increase is more likely than a decrease' 
for a particular region, if that is the best information that can be provided based on the balance of available knowledge from the Lines of Evidence Table. It is acknowledged that given the broad range of different information sources and data types (e.g., direct model output or statistical diagnostic methods) this step of the process may require some degree of expert judgement to be used.

$>$ The Projection Likelihood Information can include confidence assessment information, such as based on the degree of evidence and agreement from the Lines of Evidence table. For example, estimates of the $10^{\text {th }}$ and $90^{\text {th }}$ percentile range of plausible change is one measure that could be used to help indicate the degree of confidence in a projected future change, as well as noting wording systems used in IPCC that have a range of quantitative probabilities associated with them (Mastrandrea et al. 2011).

\section{APPLICATION OF METHOD FOR EXTREME BUSHFIRES}

For this study, the standardised method is applied for the projected change in climate from the time period 1986-2005 (i.e., a commonly used historical reference period for CMIP5 data (CSIRO \& BoM 2015)) to the time period 2040-2059 (i.e., a time period centred on the middle of this century around 2050), with a focus on summer, as requested by energy sector stakeholders for this research. The information collected here for Step 1 for the Lines of Evidence Table is intended to be relevant for the National Energy Market (NEM) region around southeast Australia, including listing any regional variations that might be important to consider, noting results presented here are derived from work done recently for energy sector applications.

The RCP8.5 scenario, representing a high emissions pathway for anthropogenic greenhouse gases, is used here for the future projections for a number of reasons. Of the set of modelled greenhouse gas emission pathways provided in CMIP5 (which start to deviate from each other after 2005), observed climate change trends for temperature indicate that the high emissions pathway RCP8.5 has been followed more closely than low emissions pathways (e.g., RCP2.6) (Schwalm et al. 2020). Additionally, although there is potential for reductions in greenhouse gas emissions and the associated rate of temperature increase later this century, RCP8.5 is used here for the application of this method given that it takes many years after changes in emissions for an emergent change in a climate trend, noting the focus for this application on the period from now until around the middle of this century. However, for applications in which projections are needed based on lower emissions pathways than RCP8.5, methods could be used for scaling these projected changes according to the global warming magnitude for a particular time period or emissions pathway (NESP 2020).

Here we examine projections of the 10-yr ARI of daily FFDI from the available modelling approaches based on GCMs, CCAM, BARPA and NARCliM. These datasets all have QME calibration applied to the input variables for each individual model prior to calculating the FFDI, with the ARI values then calculated from the FFDI data using a generalised extreme value (GEV) approach. The results show increases in the severity of fire weather conditions projected from the historical climate to the future projected climate during summer (i.e., DJF), as represented by the 10-yr ARI value of daily FFDI (Fig. 1). Some variation is apparent between the different model ensembles in the magnitude of increases, with somewhat larger increases for NARCliM in some regions, but with general agreement over these modelling approaches on projected future increases.

Extreme daily fire weather (10-yr ARI)

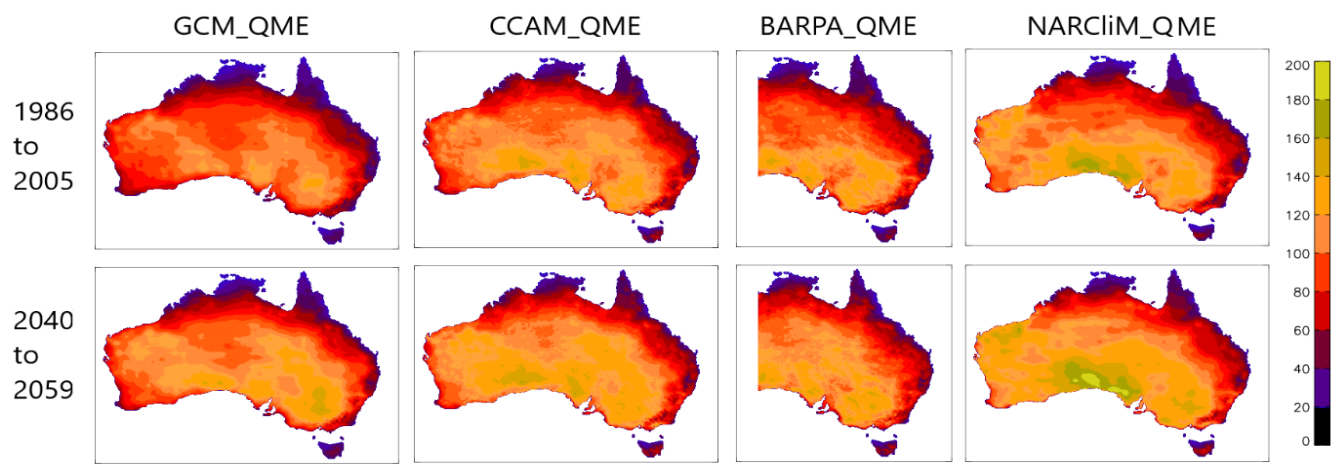

Figure 1. Projections for values corresponding to the 10-year ARI for daily fire weather conditions as represented by the FFDI. This is shown based on GCMs (left panels), CCAM (second to left panels), BARPA (second to right panels) and NARCliM (right panels), all calibrated using the QME method. Model ensemble averages are shown for historical period (1986-2005; upper panels) and future simulated climate (2040-2059 under a high emissions pathway RCP8.5 from CMIP5; lower panels). 
In addition to projections data from modelling such as shown above based on the FFDI, a wide range of other lines of evidence can also be considered for helping produce the projections information provided to end users, including for confidence assessment. Examples of such information are presented in Table 1, noting that this is derived from a large amount of content based on extensive literature reviews, with details on each line available in a larger project technical report: Dowdy et al. (2021). These lines of evidence include reviews on a wide range of physical processes, historical climate (from observations and reanalysis data) and future climate (from multiple modelling approaches in addition to, and including, Fig. 1) with additional factors also noted on lightning and fuel conditions.

Table 1. Lines of Evidence Table for extreme fire weather conditions, with a focus on summer in regions around southern and eastern Australia. In the second column, the degree of influence is listed in black, followed by whether this information implies an increase (red), decrease (blue) or little change (black) in the frequency and severity of extreme fire weather conditions, as well as by increased uncertainty (purple) in the direction of change. The rows of information are not in order of importance. Confidence information is noted for some cases when available. Additional factors are noted on lighting and fuel conditions.

\begin{tabular}{|c|c|}
\hline \multicolumn{2}{|l|}{ Physical processes } \\
\hline Individual weather factors & $\begin{array}{l}\text { Strong influence. More extreme temperatures and heatwaves; lower relative humidity; } \\
\text { small decrease in wind speed. }\end{array}$ \\
\hline $\begin{array}{l}\text { Drought and fuel } \\
\text { moisture }\end{array}$ & $\begin{array}{l}\text { Strong influence. Projected increase in frequency of meteorological drought and dry fuel } \\
\text { conditions. Considerable uncertainties for some factors; regional models likely add value. }\end{array}$ \\
\hline $\begin{array}{l}\text { Combined near-surface } \\
\text { weather conditions, FFDI }\end{array}$ & $\begin{array}{l}\text { Strong influence. Projected increase in frequency and severity of dangerous conditions in } \\
\text { general based on numerous studies; poor agreement between models near east coast. }\end{array}$ \\
\hline $\begin{array}{l}\text { Combined near-surface } \\
\text { weather conditions, FWI }\end{array}$ & $\begin{array}{l}\text { Strong influence. Projected increase, but not statistically significant, and only based on one } \\
\text { study. }\end{array}$ \\
\hline $\begin{array}{l}\text { Upper-level conditions, C- } \\
\text { Haines }\end{array}$ & $\begin{array}{l}\text { Strong influence (including extreme pyroconvection). Increased frequency of dangerous } \\
\text { conditions in southeast (including simultaneous occurrence with dangerous near-surface } \\
\text { conditions) and decrease in northeast. }\end{array}$ \\
\hline Subtropical ridge & Moderate influence in southeast. Potential increase in intensity. \\
\hline Blocking & Moderate influence. Future change uncertain. \\
\hline Fronts & Moderate influence. Future change uncertain. \\
\hline $\begin{array}{l}\text { El Niño-Southern } \\
\text { Oscillation }\end{array}$ & $\begin{array}{l}\text { Strong influence. Uncertain future change; potentially more frequent strong ENSO events } \\
\text { but with low-medium confidence. }\end{array}$ \\
\hline Indian Ocean Dipole & $\begin{array}{l}\text { Strong influence. Uncertain future change; potentially more frequent strong IOD events but } \\
\text { with low-medium confidence. }\end{array}$ \\
\hline Southern Annular Mode & $\begin{array}{l}\text { Strong influence in central east. Positive trend in SAM reducing dangerous fire weather in } \\
\text { central east region with medium confidence. }\end{array}$ \\
\hline \multicolumn{2}{|l|}{ Historical climate } \\
\hline Seasonal cycle & Models reproduce the seasonal cycle and spatial variability well (high confidence). \\
\hline Historical trend & $\begin{array}{l}\text { Increase from observations (medium confidence). Models reproduce the trend well } \\
\text { (medium confidence). }\end{array}$ \\
\hline \multicolumn{2}{|l|}{ Future climate } \\
\hline GCMs: CMIP5 & Increase (very high confidence). \\
\hline RCM: CCAM & Increase (high confidence in general; medium near east coast). \\
\hline RCM: NARCliM & Increase (high confidence in general; medium near east coast). \\
\hline RCM: BARPA & Increase (high confidence in general; based on one model to date). \\
\hline \multicolumn{2}{|l|}{ Additional factors } \\
\hline Lightning ignitions & Strong influence. Future change very uncertain, but increase more likely than decrease. \\
\hline Fuel load and type & $\begin{array}{l}\text { Strong influence. Future change very uncertain, but increased fuel load more likely than } \\
\text { decrease. }\end{array}$ \\
\hline
\end{tabular}

\section{SYNTHESIS AND CONCLUSIONS}

The Lines of Evidence Table shows considerable agreement on more dangerous fire weather conditions in a warming climate for Australia, including in relation to 10-year ARI fire weather conditions in regions around southern and eastern Australia during summer (as is a key focus here). Although there are some physical processes noted that add uncertainties, particularly based on GCM projections data, the RCM approaches can 
help with the simulation of some of these processes such that the considerable level of agreement between RCM approaches (particularly in southern Australia, but somewhat less so in parts of eastern Australia) helps add some confidence for projected future changes.

Observed trends and RCM simulations are available for near-surface and higher-level conditions, including combining those different levels using a compound event framework (Dowdy \& Pepler 2018; Di Virgilio et al. 2019; Dowdy et al. 2019), showing increases in southern Australia with more variation between results in eastern Australian including decreases in some regions. Additionally, although there is low confidence for projected future changes in vegetation-related conditions such as fuel load and type, as well as in ignition risk factors including the occurrence of dry lightning, there is some indication that increases may be more likely than decreases in risk factors associated with fuel condition and ignition sources for bushfires (while noting considerable uncertainties and more research needed on such topics).

Considering all of the review details in the sections above, and noting the predominance of an increase from the Lines of Evidence Table, projections for 10-year ARI extreme fire weather conditions in 2050 are developed here based on combining data from several calibrated modelling approaches including GCMs (4 ensemble members), CCAM (5 ensemble members), BARPA (1 ensemble member) and NARCliM (6 ensemble members). FFDI data are available from these models and are the primary data source used here. The contrasting modelling approaches are combined based on equally weighting the changes.

The ensemble median is used as a central estimate of the most probable projected change (Fig. 2). As an estimate of the range of plausible values, the second lowest value from the ensemble is used for the $10^{\text {th }}$ percentile and the second highest value is used from the ensemble is used for the $90^{\text {th }}$ percentile, with these values calculated individually at each grid cell location. However, given some of the uncertainties from Table 1 in purple (also noting some lines of evidence in blue for reductions), including variations between different modelling approaches and studies (e.g., projections based on the FWI showing smaller changes than for FFDI), the lower bound of the range provided here has been modified to reflect the potential for lower values. This is done based on reducing any projected increases for the $10^{\text {th }}$ percentile by a factor of two (as a qualitative estimate based on expert judgement). For example, at a given grid-cell location, if the $10^{\text {th }}$ percentile for the future period was higher by a value of 8 as compared to the 1986-2005 value, it would be changed to only be a value of 4 higher than the 1986-2005 value at that location. Projections for any regions that show decreases for the $10^{\text {th }}$ percentile are not changed. Only the $10^{\text {th }}$ percentile is changed to allow for lower values, but no lines of evidence suggest these FFDI projections data systematically underestimate future increases such that the $90^{\text {th }}$ percentile is unchanged and is considered a plausible upper estimate for the future projected changes for these fire weather conditions.

Based on this assessment of a broad range of factors that can influence the occurrence of extremely dangerous fire weather conditions, there is high confidence in southern Australia and medium confidence in parts of eastern Australia for the projected direction of change, with a future increase in 10-year ARI fire weather conditions being likely (i.e., 66-100\% probability) for southern and eastern Australia.

\section{Extreme daily fire weather (10-yr ARI)}

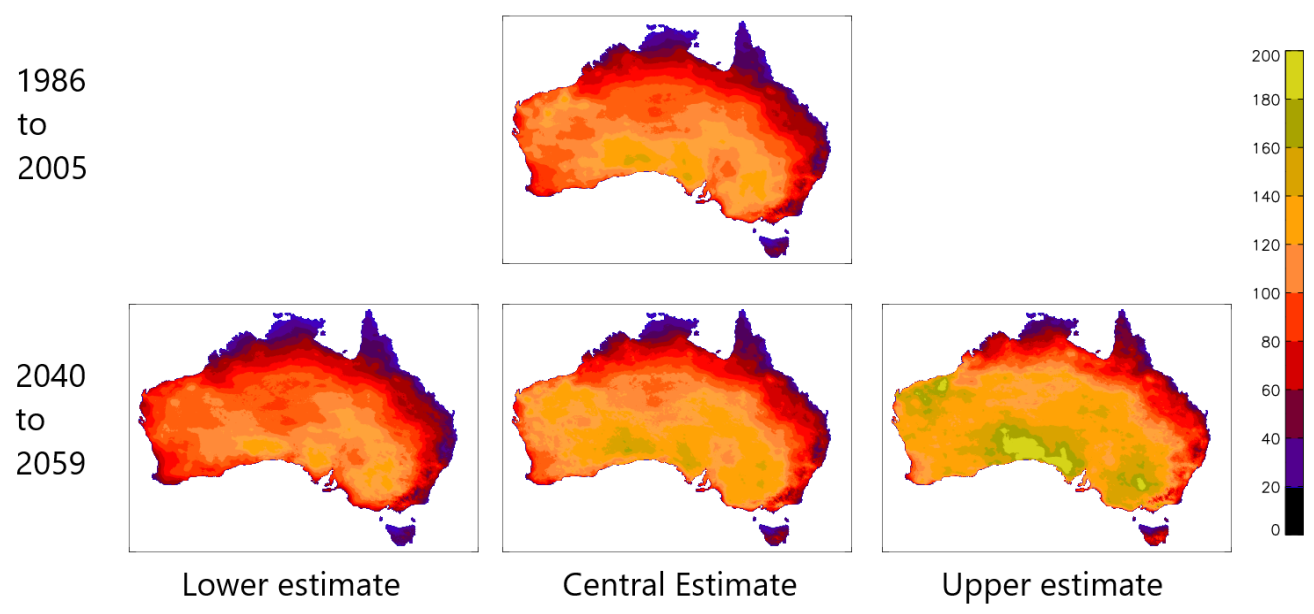

Figure 2. Projected change in values corresponding to the 10-year ARI for daily fire weather conditions during summer. Maps are shown for Australia for the historical period (based on 1986-2005; upper panel), as 
well as for the future simulated climate (based on 2040-2059 under a high emissions pathway RCP8.5: lower panels) including a central estimate with lower and upper estimates also provided. The data are based on the FFDI, with some modifications based on considering Table 1 lines of evidence.

\section{REFERENCES}

CSIRO and Bureau of Meteorology, 2015. Climate Change in Australia Information for Australia's Natural Resource Management Regions: Technical Report, CSIRO and Bureau of Meteorology, Australia.

Di Virgilio, G., Evans, J.P., Blake, S.A., Armstrong, M., Dowdy, A.J., Sharples, J., McRae, R., 2019. Climate change increases the potential for extreme wildfires. Geophysical Research Letters, 46, 85178526.

Dowdy, A.J., Pepler, A., 2018. Pyroconvection risk in Australia: Climatological changes in atmospheric stability and surface fire weather conditions. Geophysical Research Letters, 45(4), 2005-2013. https://doi.org/10.1002/2017GL076654

Dowdy, A.J., Ye, H., Pepler, A., Thatcher, M., Osbrough, S.L., Evans, J.P., Di Virgilio, G., McCarthy, N., 2019. Future changes in extreme weather and pyroconvection risk factors for Australian wildfires. Scientific Reports, 9(1), 1-11. https://doi.org/10.1038/s41598-019-46362-x

Dowdy, A.J., 2020. Seamless climate change projections and seasonal predictions for bushfires in Australia. Journal of Southern Hemisphere Earth Systems Science, 70(1), 120-138. https://doi.org/10.1071/ES20001

Dowdy, A.J., Brown, A., Pepler, A., Thatcher, M., Rafter, T., Evans, J. Ye, H., Su, C., Bell, S., Stassen, C, 2021. Extreme temperature, wind and bushfire weather projections using a standardised method. Bureau Research Report 55, Bureau of Meteorology, Melbourne, Australia. ISBN 978-1-925738-32-2

Evans, J.P., Ji, F., Lee, C., Smith, P., Argüeso, D., Fita, L., 2014. Design of a regional climate modelling projection ensemble experiment-NARCliM. Geoscientific Model Development, 7(2), 621-629.

Field, R.D., et al. 2015. Development of a global fire weather database. Natural Hazards and Earth System Sciences, 15(6), 1407-1423.

Mastrandrea, M.D., et al. 2011. The IPCC AR5 guidance note on consistent treatment of uncertainties: a common approach across the working groups. Climatic Change, 108(4), 675-691.

McArthur, A.G., 1967. Fire behaviour in eucalypt forests. Forestry and Timber Bureau, No 107, Canberra, Australia.

Mills, G.A., McCaw, W.L., 2010. Atmospheric stability environments and fire weather in Australia: extending the Haines Index. CAWCR Technical Report, Bureau of Meteorology, Melbourne, Australia.

NESP, 2020. Earth Systems and Climate Change Hub. 2020. Scenario analysis of climate-related physical risk for buildings and infrastructure: climate science guidance. Technical report by the National Environmental Science Program (NESP) Earth Systems and Climate Change Science (ESCC) Hub for the Climate Measurement Standards Initiative, ESCC Hub Report No.21, ISBN: 978-0-6489444-1-6.

Schwalm, C.R., Glendon, S., Duffy, P.B., 2020. RCP8. 5 tracks cumulative CO2 emissions. Proceedings of the National Academy of Sciences, 117(33), 19656-19657.

Su, C.-H., Ye, H., Dowdy, A.J., Pepler, A., Stassen, C., Brown, A., Tucker, S.O., Steinle, P.J., 2021. Towards ACCESS-based regional climate projections for Australia. Bureau Research Report 57, Bureau of Meteorology, Docklands, Victoria, Australia, ISBN 978-1-925738-33-9.

Thatcher, M., McGregor, J.L., 2011. A technique for dynamically downscaling daily-averaged GCM datasets using the conformal cubic atmospheric model. Monthly Weather Review, 139(1), 79-95.

Thatcher, M., et al. (2021). Technical Report on downscaling for the ESCI project. CSIRO and BoM, Victoria, Australia. Available from: https://www.climatechangeinaustralia.gov.au/en/projects/esci/

Van Wagner, C. E. (1974). Structure of the Canadian forest fire weather index, Vol. 1333, Ontario: Environment Canada, Forestry Service.

Van Oldenborgh, G.J., Krikken, F., Lewis, S., Leach, N.J., Lehner, F., Saunders, K.R., van Weele, M., Haustein, K., Li, S., Wallom, D. and Sparrow, S. (2021). Attribution of the Australian bushfire risk to anthropogenic climate change. Natural Hazards and Earth System Sciences, 21(3), 941-960. 